\title{
HACIA UN MODELO EXPLICATIVO DEL PROCESO DE ALFABETIZACIÓN EN ESCRITURA ACADÉMICA EN LAS LICENCIATURAS EN HISTORIA Y BIOLOGÍA*
}

\author{
TOWARDS AN EXPLANATORY MODEL OF THE PROCESS OF \\ LITERACY IN ACADEMIC WRITING IN THE UNDERGRADUATE \\ PROGRAMS OF HISTORY AND BIOLOGY
}

\author{
MARISOL VELÁSQUEZ RIVERA \\ Pontificia Universidad Católica de Valparaíso \\ marisol.velasquez@pucv.cl
}

\section{JUANA MARINKOVICH RAVENA}

Pontificia Universidad Católica de Valparaíso juana.marinkovich@pucv.cl

\section{RESUMEN}

La alfabetización en escritura académica se ha transformado en un desafío para las instituciones de educación superior, en especial, en Latinoamérica. En un primer momento, surgen variadas experiencias que dan cuenta de esta preocupación tanto en propuestas didácticas y metodológicas como también en investigaciones. En lo relativo a las últimas, en el presente estudio se intenta avanzar hacia una consolidación de diversos resultados ya validados en proyectos anteriores. De este modo, el objetivo que orienta este trabajo es proponer un modelo explicativo que dé cuenta del proceso de alfabetización en escritura académica según una serie de insumos que concurren en la formación de las licenciaturas en Historia y Biología de una universidad del Consejo de Rectores de Chile (CRUCH). El marco metodológico para lograr este objetivo se inserta en el paradigma cualitativo y en un enfoque interpretativo de los datos recabados a partir de grupos focales a estudiantes y entrevistas en profundidad a profesores, lo que se complementó con los programas de estudio, el correspondiente perfil de egreso y las correspondientes actividades de escritura y sus materiales asociados. Este cúmulo de datos se analizó mediante la técnica de análisis de contenido, lo que finalmente se plasma en sendos modelos explicativos en que se visualizan los hitos más importantes en el aprendizaje de cada ámbito del saber y la incidencia de la escritura en este proceso. A modo de síntesis, los modelos evidencian tres momentos

* En el marco del Proyecto Fondecyt/Conicyt 1151171 "La construcción dialógica del razonamiento en Biología e Historia a través de la escritura académica". 
esenciales en su consecución, se articulan en torno a ciertos ejes fundamentales y si bien comparten habilidades cognitivas, no sucede lo mismo con las exigencias de cada tipo de pensamiento (histórico o científico), ni con los géneros discursivos que circulan en cada comunidad disciplinar.

Palabras clave: Alfabetización académica, escritura epistémica, pensamiento histórico, pensamiento científico.

\section{ABSTRACT}

Academic literacy in academic writing has become a challenge for institutions of higher education, specially in Latin America. Initially, varied experiences arise that reflect this concern not only in terms of didactic and methodological proposals, but also in terms of research. As regards the latter, the present study attempts to move towards a consolidation of various results already validated in previous projects. Thus, the objective that guides this work is to propose an explanatory model that accounts for the process of literacy in academic writing according to a number of inputs which contribute to the formation of the undergraduate programs in History and Biology of a university belonging to the Rectors' Council of Chile (CRUCH). The methodological framework to achieve this objective is inserted into the qualitative paradigm and an interpretative approach of data collected from focus groups with students and in-depth interviews with teachers, all of which was complemented with the programs of study, the corresponding graduate profiles, and the corresponding writing activities with their associated materials. This wealth of data was analyzed using the technique of content analysis and subsequent categorization, and it is finally reflected in two explanatory models in which the most important landmarks are displayed concerning the learning of every field of knowledge and the impact of writing in this process. To summarize, the models show three essential moments in their attainment, they are articulated around certain key areas, and, while they share cognitive skills, they do not share the requirements of each type of thinking (historical or scientific), nor the genres circulating in each disciplinary community.

Keywords: Academic literacy, epistemic writing, historical thinking, scientific thought.

Recibido: 25.04.2016. Aceptado: 24.09.2016.

\section{INTRODUCCIÓN}

T a alfabetización en escritura académica se ha transformado en un desafío para las instituciones de educación superior, en especial, en Latinoamérica. En un primer momento, surgen variadas experiencias que dan cuenta de esta preocupación tanto en propuestas didácticas y metodológicas como también en investigaciones que abarcan distintos focos. En lo relativo a las investigaciones, éstas se han 
centrado, entre otras problemáticas, en dar cuenta del papel que desempeña la escritura en la alfabetización académica en los diversos contextos disciplinares. Esto en el sentido de reconocer la potencialidad de la función epistémica de la escritura que permita a los estudiantes iniciar el proceso de inserción en los distintos programas de formación universitaria y, de este modo, apropiarse del conocimiento y transformarlo.

Una serie de investigaciones realizadas en Chile ha aportado en la línea anteriormente señalada, particularmente, aquéllas llevadas a cabo por un grupo de la Escuela Lingüística de Valparaíso. Lo hasta aquí logrado es el resultado de la naturaleza cualitativa y ecológica de la investigación, considerando como unidad de análisis la comunidad disciplinar y con ello, los discursos que allí se gestan. Entre los trabajos destacan las representaciones sociales de docentes y estudiantes acerca de la escritura académica y de los géneros discursivos que circulan en las comunidades de las licenciaturas en Historia, Arte, Bioquímica y Matemáticas (Marinkovich, Velásquez y Córdova, 2012), lo que se sintetiza en un modelo comprensivo de la relación existente entre objeto de estudio, método y géneros discursivos indispensable a la hora de poner en práctica la función epistémica de la escritura (Marinkovich y Córdova, 2014). A esto se agrega la caracterización de los niveles de escritura desde lo reproductivo a lo crítico en los textos escritos por los estudiantes de las licenciaturas en Biología e Historia, así como también la constitución de perfiles de alfabetización académica derivados de los niveles, como también las modalidades discursivas, en especial, el rol de la argumentación y los rasgos lingüísticos presentes en estos mismos textos (Marinkovich, Velásquez y Olivares, 2013; Marinkovich y Poblete, 2014).

A partir de lo ya investigado, el mismo equipo ha decidido profundizar en los resultados ya obtenidos y ponerlos al servicio de un objetivo más integrador. Es así que se pretende configurar un modelo explicativo que dé cuenta del proceso de alfabetización en escritura académica proveniente del discurso de profesores y de estudiantes como también de otros insumos que concurren en la formación de las licenciaturas en Historia y Biología. En otras palabras, las entrevistas semiestructuradas a profesores, los grupos focales en que participaron sus estudiantes, los programas de estudio, el perfil de egreso y las actividades de escritura se interrelacionan en dicho modelo que se lleva a cabo según las particularidades de las disciplinas en cuestión, ambas insertas en el currículum de formación de pregrado de una universidad del Consejo de Rectores de Chile (CRUCH).

$\mathrm{Al}$ respecto, cuando proponemos un recorrido, nos estamos refiriendo a la lógica del desarrollo del conocimiento que le es propio a las disciplinas estudiadas en el ámbito académico que nos convoca. En síntesis, una lógica que responda, en definitiva, al desarrollo del tipo de pensamiento y a la metodología de indagación que les subyace. En este sentido, el objetivo último de las comunidades mencionadas es lograr que el estudiante se alfabetice académicamente y para ello la función 
epistémica de la escritura es fundamental.

En relación a lo expresado anteriormente, se establece, en primer lugar, un marco de referencia acerca de lo que significa la alfabetización en escritura académica y cómo ésta es una herramienta para desarrollar, por un lado, la alfabetización histórica y, por otro, la alfabetización científica, respectivamente. En segundo lugar, se describe el marco metodológico que sirve de sustento al estudio propuesto y se presenta el análisis de los datos recogidos de las diferentes fuentes de información, lo que permite visualizar las instancias de aprendizaje de cada disciplina y su representación en un modelo explicativo desde una perspectiva cualitativa.

\section{MARCO DE REFERENCIA}

El concepto de alfabetización académica se ha ido complejizando a medida que el interés por su implementación en el contexto universitario se hace cada vez más urgente. Radloff y De la Harpe (2000) definen la alfabetización académica como el proceso por el cual se llega a pertenecer a una comunidad científica y/o profesional, en virtud de haberse apropiado de sus formas de razonamiento, instituidas por medio de ciertas convenciones del discurso. Este concepto pone también de relieve la necesidad de que los académicos reconozcan la relación de la enseñanza de las prácticas letradas (comprensión y producción de textos escritos) con la construcción de saberes específicos de cada disciplina que se cultiva en el ámbito universitario (Romero, 2000).

Por su parte, Barton, Hamilton e Ivanovic (2000) abogan por subsumir bajo la denominación New Literacies tres componentes esenciales de la alfabetización académica, a saber, la mente, el contexto y el lenguaje, trilogía entendida como múltiples dimensiones de prácticas alfabetizadas situadas. Desde esta visión, la escritura supone procesos cognitivos que se ponen en práctica en un contexto académico determinado o, más precisamente, en una comunidad de aprendizaje específica, como mediadora en los procesos de construcción del conocimiento, cumpliendo, de este modo, la función epistémica que le es propia.

Resulta importante no perder de vista que la escritura epistémica es un constructo que no sólo se entiende desde una perspectiva cognitiva, sino también desde la cognición situada, hecho que la ubica en espacios sociales y culturales. Camps (1997) amplía esta afirmación y señala:

"El uso de la lengua es una actividad compleja inscrita en el entramado social de prácticas comunicativas y representativas. En este entramado el individuo construye su conocimiento y su pensamiento a partir de esta relación con los demás. Así, pues, la comunicación oral y escrita implica procesos sociales y cognitivos, así como procesos afectivos inseparables unos de otros" (p. 24). 
Lea y Street (2006) plantean que la alfabetización en contextos académicos puede ser vista como las habilidades cognitivo-lingüísticas, la socialización académica relacionada con la aculturación de los estudiantes en los géneros discursivos y la multiliteracidad. Estas últimas se identifican con la construcción de significados, la identidad, el poder y la autoría, lo que permitiría situarse en un nivel epistemológico determinado y en una esfera particular de actividad.

Por su parte, Carlino (2013) señala la necesidad de actualizar el concepto de alfabetización académica ampliando su cobertura a

"las acciones que deben implementarse a nivel institucional y didáctico, desde todas las cátedras, para favorecer el aprendizaje de las literacidades académicas (es decir, la participación de los alumnos en sus culturas escritas) a través de una enseñanza que las preserve como tales" (p. 372).

Ahora bien, la redefinición de Carlino toca un aspecto primordial en el ámbito académico. Nos referimos al aprendizaje de los estudiantes y su relación con quien o quienes los apoyan durante este proceso de acercamiento al conocimiento disciplinar que va a variar según las culturas disciplinares y las particularidades del lenguaje escrito que se gestan en éstas. En este proceso de aprendizaje le cabe un rol importante al tipo de pensamiento que los estudiantes deben desarrollar, sobre todo en la formación inicial de la educación superior, como es el caso de las licenciaturas en Historia y Biología que nos ocupa en este estudio.

En cuanto al concepto de pensamiento, Santiuste et al. (2001) lo conciben como el proceso que permite "responder razonadamente ante una situación relevante, poniendo en juego los recursos mentales más apropiados. Comporta un conjunto de procesos cognitivos superiores y complejos (estrategias cognitivas y metacognitivas)" (p. 47). A su vez, Larrosa (2001) agrega que tanto la lectura como la escritura marcan "una ruta de diferencias y diversidades, de silencios y palabras, de reflexiones y construcciones que nos conducen al pensamiento" (p. 8).

Desde estas menciones generales acerca del pensamiento, cabe referirse a los tipos que están involucrados tanto en el conocimiento histórico como en el científico. En efecto, el pensamiento histórico supone desarrollar una conciencia de la temporalidad, del pasado como herramienta para conocer el presente. Asimismo, se requieren capacidades relacionadas con la imaginación y la empatía históricas, destrezas para la representación histórica (desde la narración hasta la explicación causal) y habilidades para la interpretación de fuentes históricas (Monfort, Pagès y Santisteban, 2011).

Por su parte, Paul y Elder (2006) plantean que en lo relativo al pensamiento científico la resolución de problemas científicos es primordial y en ello el estudiante debe utilizar los recursos cognitivos que ya posee. Agregan que pensar científicamente es razonar acerca de conflictos, asuntos o conceptos científicos por medio 
de la aplicación de las destrezas y estructuras inherentes al pensamiento. Además, Li y Klahr (2006) declaran que el pensamiento científico es una forma de pensar sistemática, empírica y que parte de datos que son válidos. Estos últimos autores señalan que cuando el estudiante formula y prueba la hipótesis, y luego analiza la evidencia o prueba, está llevando a cabo los procesos de pensamiento científico cuya meta real es la resolución de problemas y esta instancia no hay que confundirla con la aplicación del método científico.

Finalmente, se considera que las aproximaciones conceptuales planteadas constituyen puntos de partida para comprender el pensamiento histórico como también el pensamiento científico, pilares fundamentales en el desarrollo de procesos cognitivos y metacognitivos, situados en contextos socioculturales, que se conjugan en las disciplinas ya mencionadas.

\section{MARCO METODOLÓGICO}

La presente investigación se enmarca dentro de un paradigma de carácter cualitativo. Desde esta mirada, el proceso investigativo será entendido como un intento de comprender globalmente un fenómeno o realidad estudiada y es por ello que el contenido del mismo es abordado en su totalidad. En concordancia con esto, el enfoque implementado en este estudio es de carácter fenomenológico-hermenéutico, lo que significa que los investigadores se focalizan en el reconocimiento de sucesos relevantes que respondan a los objetivos definidos para comprender el fenómeno en cuestión (Stake, 1995).

Por lo tanto, se intenta construir o reconstruir una realidad, mediante procesos de observación e interpretación, estableciendo relaciones entre todos los elementos que componen el fenómeno estudiado, tales como espacios, objetos, personas, etc. En este sentido, las investigaciones se centran en el estudio de las vivencias de las personas (Buendía, Colás y Hernández, 1998), situación que tiene directa relación con la meta del presente estudio: proponer un modelo explicativo que dé cuenta del proceso de alfabetización en escritura académica derivado del discurso de profesores (entrevistas en profundidad) y sus estudiantes (grupos focales); del análisis de sus programas de asignatura y sus actividades de escritura y del perfil de egreso de cada licenciatura.

Los estudios desarrollados bajo el alero de este enfoque responden a la implementación de un proceso comprensivo, a través del que se busca conocer patrones y conjuntos de características que permitan otorgarle una estructura o modelo al fenómeno, sin caer en la integración teórica previa, de tal manera que el proceso 
de definición de patrones o categorías no esté permeado por antecedentes conceptuales o investigativos previos, sino sólo a partir de los datos puros que emergieron de las comunidades disciplinares y discursivas investigadas.

\subsection{Fases de la investigación}

En el contexto de la investigación que nos ocupa se desarrollaron las siguientes fases: a) determinación de los objetivos de la investigación; b) establecimiento y delimitación de las unidades de análisis (unidad de contexto y unidad de registro) y parafraseo de esta última; c) establecimiento y definición de las categorías, a saber, categorías preliminares (CP) y su agrupación por relaciones de sentido, categorías generales (CG), de naturaleza más inclusiva y global; d) homogeneización de los códigos asignados (nombre consensuado de la etiqueta de cada categoría) tanto a las CP y las CG en cada entrevista, grupo focal o fuente documental, con el propósito de facilitar el análisis y la presentación de los resultados; e) análisis de frecuencia de las mismas y, finalmente, f) interpretación cualitativa de los datos y consolidación teórica, intentando dar respuestas a los objetivos de investigación.

Luego de las etapas señaladas, se implementó un proceso de revisión, lectura y análisis colectivo de la información registrada y las distintas categorías a fin de discutir en torno a posibles sesgos o situaciones de dobles interpretaciones. Una vez realizada esta acción, orientada a validar entre los investigadores la definición de códigos, se llevó a cabo la construcción de las inferencias en base a la realidad o fenómeno observado y la integración de antecedentes teóricos. Esta acción permitió recrear la realidad, transformándola de acuerdo a la interpretación realizada, a lo que se ha captado y a lo aprendido del fenómeno (Ruiz, 2003). Finalmente, la incorporación del componente teórico a la construcción de un modelo permite comprender en todos sus matices el fenómeno estudiado (Porta y Silva, 2003).

\subsection{Datos}

La información se recolectó utilizando entrevistas en profundidad o grupos focales. El uso de uno u otro dependió de la disponibilidad y/o preferencia de los entrevistados. En la Tabla I se detalla el material que se recogió según cada comunidad disciplinar. 
Tabla I. Recolección de los datos según las comunidades disciplinares investigadas.

\begin{tabular}{|l|l|l|l|}
\hline Comunidad & Método de recolección & Sujeto & Cantidad \\
\hline \multirow{2}{*}{ Historia } & Entrevista en profundidad & Profesor & 10 \\
\cline { 2 - 4 } & Grupos focales & Estudiantes & $4(8,6,5,5)$ \\
\hline \multirow{2}{*}{ Biología } & Entrevista en profundidad & Profesor & 7 \\
\cline { 2 - 4 } & Grupos focales & Estudiantes & $3(5,5,8)$ \\
\hline
\end{tabular}

Las 17 entrevistas en profundidad fueron realizadas a docentes considerados clave en la comunidad por las siguientes razones: a) dictar asignaturas de diversas líneas al interior de la comunidad, por ejemplo, Historia Universal o Biología Celular y b) haber impartido clases en dos niveles diferentes, por ejemplo, $1^{\circ}$ y $4^{\circ}$ año.

Asimismo, se consideraron siete grupos focales de estudiantes (cuatro de Historia y tres de Biología). Los cuatro de Historia corresponden a estudiantes de $1^{\circ}$, $3^{\circ}, 4^{\circ}$ y tesistas y los tres de Biología de $1^{\circ}, 3^{\circ}$ y tesistas, los que habían sido alumnos de los profesores entrevistados. Cada grupo focal contó con un promedio de seis estudiantes. De este modo, el corpus de análisis con que se cuenta corresponde a 59 participantes.

El tema central que orientó la conversación entre el entrevistador, los docentes y los estudiantes se elicitó a partir de una consigna que señalaba "cuéntenos la última vez que hizo que sus estudiantes escribieran para su asignatura”/“' ¿nos puedes contar la última vez que escribiste para la Universidad?”. A partir de esta pregunta guía, se derivaron otras del tipo ¿qué escribieron?, ¿cómo lo hicieron?, ¿cómo lo evaluaron?, ¿qué fue lo más fácil/difícil de tal actividad?, ¿pueden reconocer cambios en cuanto a la escritura de cuándo ingresaron a la universidad hasta ahora?, ¿cuáles?, etc. Todas las preguntas anteriores apuntaban a identificar, por ejemplo, los géneros que circulaban en cada comunidad, las actividades de escritura, la evaluación de la escritura, el rol del profesor en la producción escrita, las modalidades discursivas predominantes, etc., temáticas que fueron emergiendo a partir de los objetivos del proyecto al que se hizo alusión en la Introducción. Si bien previamente a cada entrevista y grupo focal se contaba con una pregunta principal $\mathrm{y}$ varias preguntas asociadas, estas últimas pudieron generar otras que permitieran aclarar, profundizar o indagar en otros focos de interés. El diseño de la entrevista se validó a partir de juicio experto.

Los datos recogidos a partir de los instrumentos señalados se complementaron con los programas de asignatura de los profesores entrevistados, las actividades de escritura que incorporan en sus clases (instrucciones, pautas, rúbricas, pruebas y/o presentaciones PPT, etc.) y el perfil de egreso de cada licenciatura. En cuanto a los 
documentos recogidos, se cuenta con 9 programas y 11 actividades de escritura).

\subsection{Técnica de análisis de los datos}

Cada una de las entrevistas y grupos focales fueron transcritos para facilitar el posterior proceso de análisis. Esto se hizo separando las intervenciones según turnos de habla. Además, se asignó un número a cada una de ellas para facilitar su posterior búsqueda en caso de que se necesite referenciar ejemplos.

Tras lo anterior, el primer procedimiento que se aplicó consistió en identificar unidades de registro dentro del corpus de análisis, es decir, aquellas partes analizables que tengan contenido significativo para los fines del estudio. En este caso, nuestro interés se encontraba en toda aquella información que se refiriera a los temas clave (géneros, actividades de escritura, rol del docente, proceso de escritura, procedimientos, evaluación, evolución de la escritura, etc.).

Para efectos de simplificar el análisis, cada una de estas unidades se parafraseó, manteniendo la información de utilidad para la investigación. Posteriormente, se dio paso al proceso de codificación, asignando categorías a las unidades de registro identificadas. Este procedimiento se denomina microanálisis y se basa en un trabajo línea por línea, aunque también es posible aplicarlo a una palabra, oración o párrafo. Por esto, es posible que una unidad de registro contenga más de una categoría según su contenido. En esta etapa se comparó de manera constante la información relevada para relacionar distintas unidades que aborden un mismo concepto, procedimiento y/o contenido, a fin de homogeneizar los datos.

En la Tabla II se presenta un ejemplo de codificación de fragmentos de entrevista siguiendo los procedimientos descritos.

Tabla II. Establecimiento de categorías preliminares a partir de las unidades de registro y parafraseo.

\begin{tabular}{|l|l|l|}
\hline Unidad de registro & Parafraseo & Categoría preliminar \\
\hline $\begin{array}{l}\text { entonces yo tomo esa instancia como } \\
\text { para retroalimentar, sugerir que debe- } \\
\text { ría ir en un artículo ya más formal }\end{array}$ & $\begin{array}{l}\text { Primera entrega: ins- } \\
\text { tancia de retroalimen- } \\
\text { tación }\end{array}$ & $\begin{array}{l}\text { Etapas del proceso de } \\
\text { escritura }\end{array}$ \\
\hline $\begin{array}{l}\text { y luego se les entrega una pauta que } \\
\text { nosotros sacamos de un libro que tie- } \\
\text { ne el profesor XXXX acerca de cómo } \\
\text { redactar un trabajo científico }\end{array}$ & $\begin{array}{l}\text { Se entrega una pauta } \\
\text { para la redacción de } \\
\text { un trabajo científico }\end{array}$ & Pauta de escritura \\
\hline $\begin{array}{l}\text { entonces ahí nosotros les damos cier- } \\
\text { tas orientaciones de lo que debería ir y } \\
\text { de qué es lo que es importante relevar } \\
\text { en cada una de las etapas, etc. }\end{array}$ & $\begin{array}{l}\text { Se dan orientaciones } \\
\text { para cada etapa del } \\
\text { escrito }\end{array}$ & $\begin{array}{l}\text { Indicaciones para es- } \\
\text { cribir }\end{array}$ \\
\hline
\end{tabular}


Este mismo procedimiento se aplicó al análisis de los documentos asociados a cada docente (programas y actividades de escritura) que se recolectaron y al perfil de egreso de cada licenciatura.

\section{CONSOLIDACIÓN DE LOS RESULTADOS}

La sección que se despliega a continuación es el resultado de un análisis exhaustivo de todas las fuentes de información que permitieron derivar categorías preliminares (CP) y generales (CG) que constituyen posteriormente la base para la consolidación teórica, plasmada en un modelo explicativo. En este último se pretende dar cuenta de la ruta que siguen los estudiantes cuando se enfrentan a los desafíos que les impone el proceso de alfabetización en escritura académica una vez insertos en las comunidades disciplinares de las licenciaturas en Historia y en Biología, respectivamente.

\subsection{Licenciatura en Historia}

En un primer momento, el centro gravitacional de la alfabetización es la Actividad de aprendizaje, eje fundamental que se mantiene durante todo el primer ciclo de inserción de los aprendientes en la comunidad disciplinar. La Actividad de aprendizaje se concibe mayoritariamente desde las actividades de lectura y luego la escritura dentro de un marco acreditativo del conocimiento, en que el aprendiente debe analizar y explicar las ideas de otros, describir situaciones y hechos, demostrar su comprensión de ciertos procesos históricos y justificar sus opiniones, usando como base las ideas de los autores. Al respecto, un estudiante señala:

"No puedo escribir algo que yo sé que leí en alguna parte, que yo sé que es verdad, pero llegar y escribirlo solo no, sino que tiene que haber una cita entre medio que me respalde; eso es como algo que no han dejado casi todos los profesores acá: ' $¡ Y$ quién dijo esto?, tiene que citarlo; porque lo inventó usted o es plagio'. Entonces son como varias cosas, la metodología y siempre el respaldo de la bibliografía y todo lo que uno hace" (HISTFoc1, 22).

El siguiente ejemplo corresponde a una pregunta aplicada en primer año.

"El acaecer de la Historia y la Historia de la cultura. Señale en qué sentido la Historia de la Cultura está contenida en el Acaecer de la Historia, suponiendo una praxis humana que reproduce el medio natural y agregando un elemento poiético de creación de la realidad. Desarrolle, fundamente con los autores y ejemplifique" (HISTPrueb1, 1). 
Si bien el Género íntimamente relacionado a la Actividad de aprendizaje es la prueba (en clases, para ser resuelta en la casa, control de lectura, etc.), siempre presenta el apelativo "tipo ensayo", es decir, el aprendiente debe expresar aunque sea un atisbo de opinión frente a las ideas de los autores. Un estudiante comenta en un grupo focal:

"Me dijeron, la prueba se responde tipo ensayo. Y la mayoría (...), por lo menos, parte relevante de los que somos el grupo de primer año no sabemos hacer un ensayo... No sabemos responder preguntas ensayísticas, porque venimos acostumbrados a un sistema de responder, aprenda a responder con alternativas. Aprenda a memorizar y demuestre conocimiento" (HISTFoc2, 18).

Asimismo, se propone el Trabajo de Investigación como instancia para promover las habilidades de búsqueda, sistematización y presentación de la información. Un estudiante declara:

"Trabajos de investigación que tienen la misma estructura de los ensayos, que va identificar un tema, un problema, una hipótesis y después los argumentos. Y si eso, por lo menos, en metodología y en ramos, son súper rígidos, rígidas también, porque el principio pasan, los ayudantes creo que pasan, un documento con la estructura que sale cómo hacer un tema, o sea, cómo identificar un tema; después de ahí sacar un problema, después cómo hacer una hipótesis, después cómo estructurar la introducción incluso, que claro, primero se presenta esto, después el tipo de fuente, la metodología, eh... las preguntas de investigación." (HISTFoc3, 93).

La Modalidad predominante entonces es la descripción y la explicación, aunque no se desconoce la presencia incipiente de la argumentación, tal como lo señala un docente:

"Tú tienes de la descripción, entonces tú le dices está bien, pero a eso hay que agregar conceptos para un mejor análisis, pero después tú puedes pasar a una tercera etapa que son el concepto (...) en algunos puedes pasar el control social, entonces tú pides que expliquen qué el mestizaje, y luego tú bajas y aplicas los conceptos desde la Historia” (HISTEnt5, 19).

Las Habilidades cognitivas que subyacen son el análisis, la síntesis, la jerarquización y categorización de la información y, especialmente, la justificación. Un estudiante señala:

"Y luego hacer un análisis a partir de eso, ya sea un contraste, no sé, a nivel social si 
es un tipo, no sé, de análisis social, presentábamos lo que decía el autor y después lo aplicábamos" (HISTFoc3, 92).

La Mediación es realizada por los ayudantes de cada curso, quienes toman controles de lectura, ayudan a comprender los textos, proponen y corrigen evaluaciones y colaboran en el proceso de acercamiento disciplinar de los aprendientes. Al respecto, un estudiante declara:

"En el caso de Grecia, en el caso de que si se pueda hacer un ensayo, el profesor a lo más da una semana y se tiene que hacer el ensayo en una semana y no hay retroalimentación directa con el profesor, a lo más puede ser con los ayudantes" (HISTFoc 3, 253).

El ayudante cumple así con colaborar en el proceso de aprendizaje de los estudiantes y constituirse como nexo entre ellos y el profesor, además de ayudar a transmitir los aspectos fundamentales de la asignatura.

En este punto es interesante señalar que los docentes están más alejados del proceso que implica acompañar a los estudiantes en sus Actividades de Aprendizaje, pues asumen que las habilidades asociadas a este primer ciclo de inserción disciplinar deberían traerlas consolidadas desde la enseñanza secundaria y que no es responsabilidad de los profesores desarrollar esos conocimientos y habilidad en sus estudiantes. Los profesores reconocen que no tienen la disponibilidad de tiempo para hacerlo, ni tampoco la formación en escritura académica.

"Y ahí se notaba que, es obvio que en algunos colegios enseñan más a hacer ensayos que en otros y eso como que uno sabe, los que vienen de buenos colegios, ya saben hacer ensayos, lo tienen que haber aprendido en la Enseñanza Media..." (HISTEnt4, 134).

Este primer momento de la alfabetización académica puede reconocerse durante los dos primeros años de la formación académica, tal como lo señala un estudiante.

"Así la forma en que te tratan cuando entras es bastante atemorizante, en algunos momentos, ya segundo y tercer año uno dice 'ya' como que se relaja, ya entiende; le pasan un ensayo y dice 'ya, de aquí al jueves, lo hago' [risas de los participantes], pero siempre en primer año como que uno no entiende como mucho cómo hacer las cosas y nadie explica” (HISTFoc1, 51).

En relación al segundo momento que se reconoce en el proceso de alfabetización en escritura académica en Historia, lo primero que es necesario señalar es que 
los límites con el anterior son difusos y no está configurado exclusivamente entre el tercer y cuarto año de la formación disciplinar, sino que se reconocen algunas actividades de aprendizaje que persiguen acercar al aprendiente a las convenciones, exigencias y particularidades de la comunidad.

El foco central en este momento intermedio lo constituye la Argumentación como modalidad discursiva predominante. La principal diferencia con el momento anterior, lo constituye el cambio de una modalidad monológica a una dialógica, la relevancia que adquiere la fundamentación por sobre la justificación -por su carácter más centrado en el uso de apoyos bibliográficos- y la mayor presencia de esta modalidad discursiva en las actividades de clases que implican la producción de textos escritos. Un estudiante señala:

"Y luego hacer un análisis a partir de eso, ya sea un contraste, no sé, a nivel social si es un tipo, no sé, de análisis social, presentábamos lo que decía el autor y después lo aplicábamos por así decirlo, o también dar a entender un punto de vista de nosotros, pero siempre respaldando lo que eran los autores, lo que se había hablado en clases, yo creo que por ahí va" (HISTFoc2, 92).

En cuanto a la Mediación, también se aprecia un cambio importante. En este segundo momento, la Mediación está entendida como Acompañamiento Situado, esto es, ya no sólo la figura del ayudante es crucial, sino que el docente adquiere un rol de guía del proceso de aprendizaje y del desarrollo de la escritura y utiliza variadas estrategias didácticas, tanto enfocadas en el proceso de escritura como en la calidad del producto. Durante la entrevista, un docente señala:

"Y también para eso los estudiantes, una semana antes de entregar el ensayo, vienen a hablar conmigo para saber en qué tema están trabajando y les doy algunas orientaciones, ya sea temáticas, metodológicas o bibliográficas, y después de la entrega del ensayo hay una pauta de evaluación también, en la que se consideran puntos como la claridad, tipo de organización del argumento, etc." (HISTEnt3, 44).

Los géneros que se escriben en este período son las pruebas -en todas sus variedades-, el paper, que implica un mayor desafío cognitivo y los ensayos, por lo que las habilidades referidas a la investigación son fundamentales. Así, la utilización analítica de fuentes es una actividad permanente y sistemática, tal como se declara en un programa de asignatura:

\section{"6.- METODOLOGÍA}

- Clases expositivas sobre mundo moderno e historiografía.

- Análisis de fuentes: lectura, análisis y problematización de textos escritos durante el período estudiado. 
- Supervisión de elaboración de ensayo de investigación.

- Aplicación de conocimientos en pruebas orales y escritas" (HISTPrograma 4).

Resulta curioso que en el primer período de inserción en la disciplina, el rol de mediación esté concentrado en la figura del ayudante y que el docente no realice actividades de apoyo intencionado a los estudiantes. Podría explicarse por la cultura de la comunidad que considera provechoso "forzar" a los estudiantes a aprender las dinámicas de la disciplina, concentrándose en la tenacidad de ellos para conseguir la aprobación de las asignaturas. Asimismo, es importante destacar que la Argumentación está en el centro de las dinámicas de la comunidad, como eje generador de aprendizajes. Un estudiante señala:

"No sé, dar la hipótesis o la idea que uno quería plantear, con tres argumentos, teniendo una introducción, una conclusión, como ese sería el... introducción, desarrollo, conclusión" (HISTFoc3, 36).

El tercer momento que se reconoce en la alfabetización en escritura académica también está centrado en la Argumentación, pero ya no sólo como respaldo de ideas, sino como Confrontación de fuentes y también como propuesta de visiones de parte de los estudiantes. Se aprecia así un marcado carácter activo y participativo de los estudiantes en los cursos más avanzados, tal como señala un alumno:

"En la introducción se plantea lo que uno propone y luego vendrían, como en ese caso yo me acuerdo, dos o tres argumentos; y luego la conclusión que tiene que recoger, sintetizar y dar, no sé, nuevas líneas de investigación, claro. Esa sería como la estructura que a mí se me viene a la cabeza" (HISTFoc2, 104).

Por lo tanto, se siguen trabajando las pruebas tipo ensayo, los trabajos de investigación de naturaleza ensayística, los ensayos propiamente tal y, más adelante, al finalizar el ciclo formativo, las tesis. Todo esto con el objetivo de desarrollar la capacidad de dialogar con las fuentes y el fomento de la postura personal, sellos indiscutibles del Programa de Licenciatura en Historia, tal como se plantea en el perfil de egreso:

"El egresado/a de la Licenciatura en Historia con mención en Ciencia Política posee las capacidades para aplicar un conjunto de conocimientos y métodos científicos que le permiten interpretar y comprender, a través de la investigación, los procesos históricos" (PUCV, 2015a).

Los ciclos, anteriormente descritos, confluyen de manera espiralizada en desarrollar la alfabetización académica en escritura en esta comunidad en particular, 
aunque este proceso no es consciente en todos los actores, pues consideran que los procesos de lectura y escritura en la universidad no son diametralmente diferentes a los que se desarrollaron en la enseñanza secundaria, desconociéndose así las particularidades de la disciplina y el sello que la comunidad le imprime a sus miembros.

A modo de síntesis, en la Figura 1 se visualizan los componentes del proceso de alfabetización en escritura académica en la Licenciatura en Historia que está elaborado -como se explicó precedentemente en el marco metodológico- según los grupos focales aplicados a estudiantes de distinto nivel de inserción en la disciplina, de las entrevistas en profundidad a los docentes y del análisis de los programas de estudio, las actividades de escritura y el perfil de egreso de dicha licenciatura:

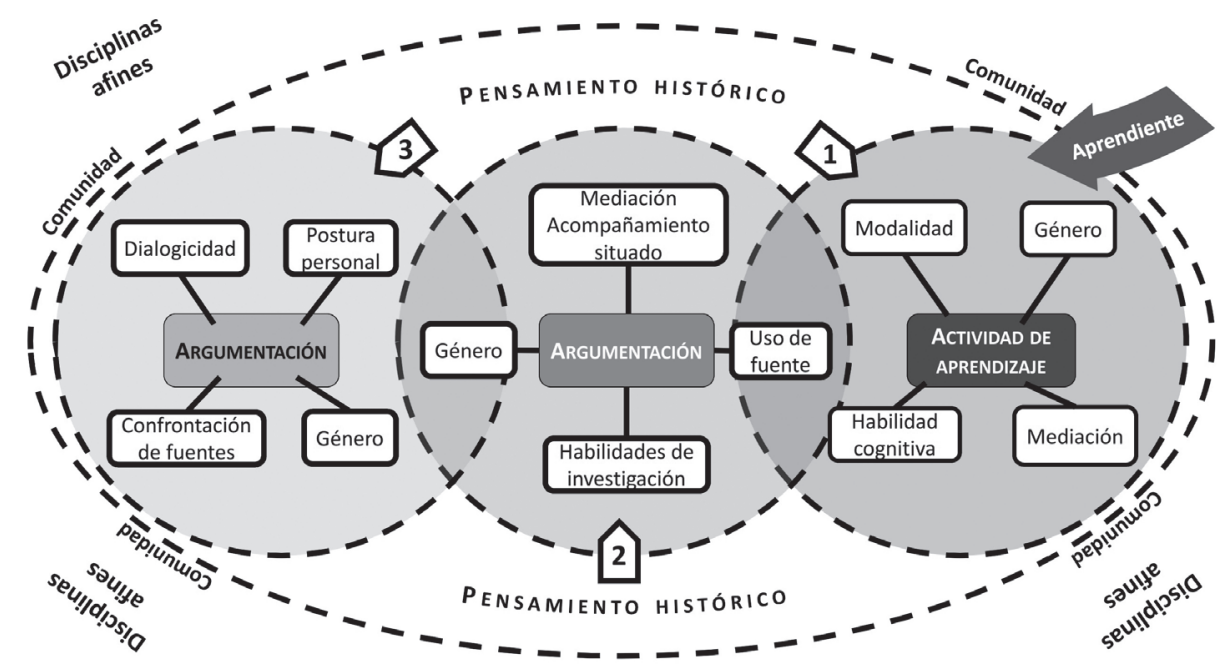

Figura 1. Modelo explicativo del proceso de la alfabetización en escritura académica en Historia.

En el modelo presentado se puede reconocer un proceso de alfabetización en escritura académica desde el momento en que el aprendiente ingresa a la comunidad disciplinar hasta que egresa de ésta, distinguiéndose claramente tres instancias que se configuran en torno a un eje central que marca y guía las restantes en una interrelación sinérgica. En todas estas instancias están presentes la lectura y la escritura como habilidades que permiten apropiarse del conocimiento disciplinar a través de los géneros y modalidades discursivas propias a esta comunidad. 


\subsection{Licenciatura en Biología}

En un primer momento, los estudiantes se enfrentan a lo que hemos llamado Aproximación científica a un problema. Esta especie de introducción o iniciación al desarrollo del Pensamiento cientifico y de la Escritura científica se concibe desde los primeros cursos a los que se enfrenta el estudiante y su objetivo principal es desarrollar la habilidad de acercamiento a la labor que realiza un biólogo, tal como lo señala un docente:

"Bueno, insisto en este tema de la aproximación científica, porque aquí en esta asignatura en particular no estamos buscando respuestas así hechas, definiciones o enumere los fenómenos asociados con tal y tal cosa o defina sobre esto. No es ese el tipo de pregunta que se hace" (BIOEnt7, 47).

Esta Aproximación científica a un problema implica el desarrollo de una serie de habilidades cognitivas (observar, definir y asociar, etc.) y la producción de géneros específicos (informe de salida a terreno e informe de Laboratorio). En una guía de trabajo se encuentra la siguiente instrucción:

"Sabiendo que el recurso semillas (y sus diferentes tamaños) es vital para la sobrevivencia de los pinzones granívoros y que se relacionan directamente con el tamaño de su pico, realice la siguiente actividad utilizando la lámina de los histogramas de distribución de tamaños de pico (...) ¿Cómo podemos relacionar los patrones del tamaño del pico del pinzón mediano en las diferentes islas con el origen de las tres especies? Genere una hipótesis sobre el origen de las especies" (Biología Fundamental, $1^{\text {er }}$ Semestre).

Sin embargo, los docentes reconocen que no se enseña a escribir en la universidad, pues, como se ha señalado anteriormente en el caso de Historia, se asume que es un conocimiento que debiera haberse aprendido en la enseñanza secundaria.

"No nos corresponde tampoco hacer esas cosas de lenguaje... creo que es responsabilidad de la Universidad que debería tomar estas cosas y creo que esto debería ser fuera del currículum, porque a nosotros nos colocan a dar un curso de inglés, nos quitan cuatro" (BIOEnt1, 28).

A pesar de lo anterior y que pudiera parecer que hay un absoluto desinterés por la lectura y la escritura en las disciplinas, se reconoce una íntima relación entre los géneros discursivos que circulan en la comunidad en la primera etapa de la formación de los estudiantes y las habilidades cognitivo-lingüísticas que se requie- 
ren para el desarrollo del pensamiento científico. La metodología indagatoria en ciencias se instala en la comunidad desde el inicio del proceso de alfabetización académica y de inserción disciplinar.

En relación al segundo momento en el proceso de alfabetización en escritura académica en Biología, Aproximación científica a la resolución de un problema, éste se configura del mismo modo que el anterior, es decir, en base al género paper y a habilidades cognitivas que implican la presencia, contraste y/o defensa de una idea personal del estudiante. En el programa de una asignatura de tercer año se señala:

"Evaluación del aprendizaje (...)

Evaluación de exposición oral y del informe escrito (paper según formato de la Revista Chilena de Historia Natural) del resultado de una investigación original sobre comportamiento reproductivo. Se evalúa: expresión oral, creatividad y pertinencia de la investigación y de la presentación, respeto y cuidado de la fauna utilizada, veracidad de la información, respeto por las opiniones de los pares, revisión bibliográfica, análisis crítico de la información, establecimiento de relaciones e integración de conceptos. Se contempla auto y coevaluación (Ponderación 20\%)" (BIOPrograma 6).

El punto que diferencia el primer momento del segundo radica en la resolución de un problema de índole científico. Si bien en ambos momentos se reconoce un proceso de inducción, acercamiento o aproximación a los constructos biológicos como modo de aculturación disciplinar; en el segundo, el foco está en la resolución de problemas en términos de una actitud propositiva del estudiante en que sea capaz de defender sus ideas. Con esto se demuestra la importancia de la argumentación en ciencias, tal como lo reconoce un docente:

"Y en un trabajo científico para ser publicado es lo clásico: una introducción que para mí tiene que ser conducente a la hipótesis. Si una introducción de alarde de conocimiento no conduce a la hipótesis, no sirve. Ahora, al conducir a la hipótesis, la hipótesis me genera el problema, me explicita el problema. Yo a eso le puedo agregar un objetivo general, que para mí es otra forma de decir la hipótesis que el rol para mí de los objetivos específicos es desglosar las hipótesis en objetivos más operacionales, por decirlo así, ¿no es cierto?, si yo quiero estudiar la relación de una planta con un insecto, bueno, yo por un lado conozco la planta, por otro lado el insecto y después los integro" (BIOEnt5, 97).

En cuanto al tercer momento del modelo propuesto, Consolidación del conocimiento cientifico, éste se considera, por una parte, ligado a los procesos de in- 
vestigación que se propician durante la elaboración de la tesis y en su posterior defensa pública. Esta consolidación no se comprende si no la relacionamos con la escritura. Un profesor lo explica así:

"Yo diría que... porque al final la escritura para mí es lo que les decía, es escrito del lenguaje... de los pensamientos. Cuando un estudiante tiene mejor nivel, tiene mejor claridad de ideas, ideas claras, conceptos claros, por lo tanto, puede aplicar todas las acciones de la taxonomía de Bloom, desde leer o conocer hasta la aplicación o la síntesis de mucha mejor manera y si ese lenguaje de ideas y conceptos, $\underline{\text { la escritura inevitablemente lo es... el lenguaje escrito yo creo que es un segundo }}$ nivel del pensamiento..." (BIOEnt6, 48).

Y, por otra parte, estrechamente relacionado con el desarrollo del pensamiento cientifico, concebido como un proceso de aculturación al interior de la disciplina, que implica un modo particular de ver, considerar y relacionarse con las prácticas científicas que coexisten entre los biólogos, tal como se reconoce en el perfil de egreso:

"El saber hacer de un Licenciado en Biología comprende la habilidad para formular hipótesis en relación al fenómeno de la vida, diseñar experimentos que las pongan a prueba, analizar, validar y discutir sus resultados, y comunicarlos en forma oral y escrita. El Licenciado en Biología debe ser capaz de aplicar el método científico, y si es de su preferencia, hacerlo con énfasis en la ciencia aplicada" (PUCV, 2015b).

A manera de síntesis de los tres momentos del modelo de Alfabetización en Escritura Académica, construido a partir de los datos recabados en la comunidad de Biología, cabe señalar que éstos se configuran en hitos o peldaños que va sorteando progresivamente el ingresante en el recorrido desde que se encuentra fuera de las fronteras de la comunidad hasta que consigue su validación, posterior al proceso de defensa de tesis. Un profesor señala al respecto:

"Yo te diría que el cambio más grande se produce cuando terminaron la tesis, (...) al final yo te diría que [el estudiante] es capaz de tener capacidad de plantear cosas propias, de defender un resultado (...) yo diría que el mayor cambio se genera al final cuando ellos pasaron por todo lo que es escribir, redactar, defender públicamente" (BIOEnt4, 90).

Además, el modelo incluye -en su formalización- el aporte de otras ciencias que circundan la disciplina de la Biología, tanto en lo que se refiere a la dimensión 
de la escritura cientifica como a la del pensamiento científico. Los miembros de la comunidad reconocen y valoran la importancia y rol de otras disciplinas, y su relación con ambas dimensiones, aunque en algunos casos la escritura se visualiza como aspectos superficiales del lenguaje. Un docente señala:

"O sea, la correlación es directa (...) hacen Matemáticas, hacen Química, hacen Física, pero con eso ya adquieren ciertos conocimientos que les empiezan a dar un lenguaje. Lo de Química se usa en Biología, lo de Matemática se usa en Biología, la Física se usa en Biología, entonces cuando llegan a los ramos biológicos, él de a poco está armando su stock, por decirlo así” (BIOEnt6, 44).

Finalmente, en el modelo presentado se reconoce que el fin último de la alfabetización en escritura académica es el desarrollo de la díada pensamiento-escritura cientificos como las motivaciones últimas de la Licenciatura en Biología. Esto se registra en el perfil de egreso:

"Competencias Específicas Disciplinares: (...)

- Comunica el conocimiento de las diferentes disciplinas de la Biología como también los resultados de la investigación científica a audiencias especializadas y no especializadas" (PUCV, 2015b).

Aún más, los mismos estudiantes reconocen no sólo las particularidades de la escritura en la disciplina, sino que también el rol que le corresponde al especialista disciplinar en el desarrollo de la alfabetización en escritura académica.

"Pero lo que pasa es que tú no le puedes dejar la parte del lenguaje al profesor de lenguaje, porque tú usas el lenguaje en Física, en Química, en Biología; de maneras distintas; entonces, o sea, desde mi óptica... te deberían enseñar a escribir en ciencias" (BIOFoc2, 74).

Considerando la descripción pormenorizada de las instancias del proceso de alfabetización en escritura académica, según lo planteado por las distintas fuentes de información recopiladas y analizadas en el marco de la Licenciatura en Biología, en la Figura 2 que se presenta a continuación se propone un modelo explicativo de cómo la comunidad disciplinar se representa dicho proceso: 


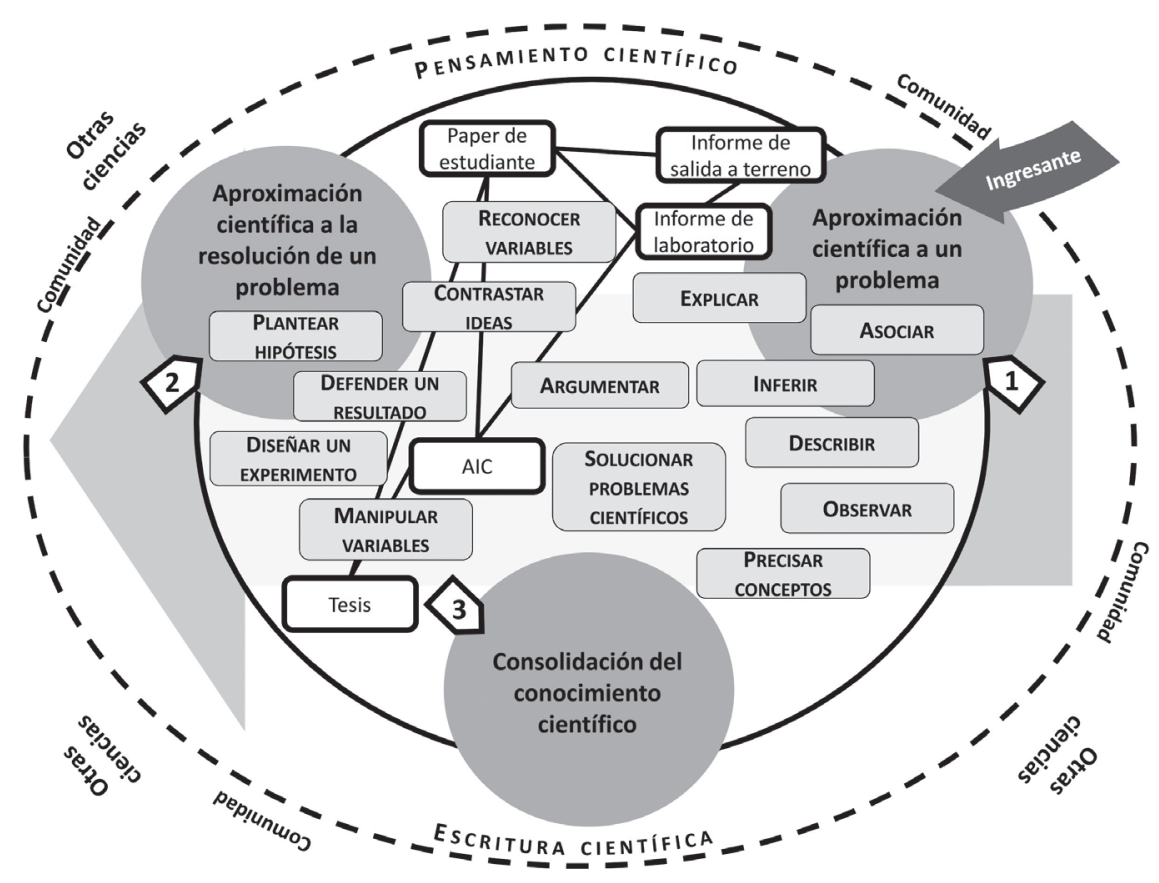

Figura 2. Modelo explicativo del proceso de alfabetización en escritura académica en Biología.

En este modelo se constatan, tal como lo anunciáramos en Historia, tres momentos, aunque el último es la consecuencia de los dos primeros. El foco de estos momentos es la resolución de un problema de orden científico, que supone una serie de habilidades cognitivo-sociales y discursivas, que se encuentran plasmadas en los géneros que circulan en esta comunidad según las necesidades de cada nivel de alfabetización académica.

\section{CONCLUSIONES}

Dialogar con las comunidades disciplinares en la universidad en torno a la problemática de la alfabetización en escritura académica significa, para los fines de esta investigación, situarse principalmente desde una perspectiva cualitativa que supone adentrarse en las licenciaturas en Historia y en Biología. De este modo, interesa comprender las múltiples realidades de las comunidades investigadas para describirlas, interpretarlas y luego construir una síntesis de lo logrado. 
En este contexto, los resultados obtenidos a partir de las etapas de la investigación realizada se plasman en sendos modelos explicativos que dan cuenta de la ruta de aprendizaje por la que transitan los estudiantes, acompañados por los docentes y/o ayudantes, cuando se aproximan al conocimiento disciplinar en las licenciaturas ya nombradas. En las gráficas de los modelos se observan tres momentos o hitos que señalizan la ruta de cada campo disciplinar circunscrito dentro del tipo de pensamiento, sea histórico o científico, asociados a algunos ejes que se relevaron como fundamentales en el discurso de los actores o de la declaración en los documentos recopilados.

Los momentos evidenciados en los modelos son en cantidad equivalentes y con límites difusos con respecto a los niveles de formación, sin embargo, se reconoce una progresión en el desarrollo de la alfabetización en escritura académica, tanto en Historia como en Biología. Asimismo, en ambas disciplinas se aprecia una especie de doble estándar respecto de dicho proceso: los docentes se refieren a que no es responsabilidad de ellos enseñar a leer y escribir en la universidad, pues los estudiantes debieran traer ese conocimiento desde la enseñanza secundaria; sin embargo, a partir de las acciones que se realizan en la comunidad, se reconoce que tienen un proceso de acompañamiento al ingresante en la comunidad, aunque no es del todo explícito y reconocido por todos los miembros.

Este último hallazgo resulta interesante en el sentido de compartirlo con las comunidades estudiadas ante la posibilidad de poner en práctica estrategias de acompañamiento que facilitarían una inserción más natural tanto de los nuevos miembros de la comunidad como de aquellos que han ingresado precedentemente, cumpliendo así con el rol formador que le compete a todo miembro de la comunidad universitaria.

En cuanto al desarrollo del pensamiento, si bien se reconocen diferencias en el énfasis en las habilidades cognitivas según cada disciplina, las modalidades discursivas, los géneros que circulan en las comunidades en cuestión y las actividades de escritura, no es menos cierto que ambas comunidades tienen como fin último la formación del pensamiento crítico como parte fundamental del proceso de alfabetización académica y, por tanto, del rol que le compete a las prácticas letradas en dicha formación.

Ahora bien, en Historia, en lo relativo a la alfabetización en escritura académica y el desarrollo del pensamiento histórico, es posible concluir que tanto el discurso de docentes y de estudiantes como de los documentos revisados están ligados a la resignificación del pasado a través del uso deliberado de ciertos focos de análisis, especialmente en lo que se refiere al desarrollo del tiempo histórico, la causalidad, la objetividad y la intertextualidad. Cada uno de éstos se representa con unos rasgos escriturales específicos y están asociados a modalidades discursivas determinadas. Por ejemplo, en el desarrollo del tiempo histórico se privilegia una modalidad narrativa por sobre una argumentativa, a diferencia de la intertextua- 
lidad en que dialogan las fuentes y, por tanto, se contrastan, discuten y defienden ideas. Al respecto, Plá (2005) plantea que la escritura contribuye a la focalización de estos ejes analíticos que sumados configuran el pensamiento histórico. Este planteamiento es compartido por investigadores del área que señalan que el pensamiento histórico no es un conocimiento intuitivo y natural, sino que requiere un proceso formativo gradual (Sáiz y López-Facal, 2015).

Por su parte, en Biología, a diferencia de Historia, luego del análisis de las fuentes de información mencionadas anteriormente, el proceso de alfabetización en escritura académica y el desarrollo del pensamiento científico están fuertemente relacionados con la resolución de un problema de orden científico para lo cual el ingresante debe seguir un recorrido sistemático y basado en datos que debe someter a prueba. En este proceso, las prácticas escriturales están presentes a través de las modalidades discursivas de la descripción, la explicación y la argumentación; esta última prima, especialmente, durante la formulación de la hipótesis como parte importante de la experimentación y de la defensa de los resultados obtenidos. Es esencial plantear, al respecto, el valor de la escritura epistémica relacionada con la especificidad de la escritura científica y, por tanto, con la alfabetización científica a la que se ven enfrentados aquellos estudiantes que aspiran a convertirse en licenciados y, más adelante, en biólogos. Se reafirma así lo que ya Lemke (1990) manifestaba cuando se refería a la noción de hablar ciencia como hacer ciencia a través del lenguaje en las instancias, por ejemplo, de observar, comparar, evaluar y decidir frente al estudio de un fenómeno científico.

El hecho de haber podido expresar, esperamos, la riqueza de los datos tal como los recogimos desde las fuentes de información in situ supone, por una parte, un aporte invaluable tanto para el conocimiento de los mismos actores como para las comunidades involucradas en lo que se refiere a la construcción de los significados disciplinares y en la incidencia de la escritura en dicho proceso. No menos importante es la posibilidad de replicar esta experiencia con otras disciplinas del currículum universitario en las que ya hemos incursionado y, aún más, profundizar en las prácticas letradas en los distintos escenarios áulicos.

Sin lugar a dudas, investigar la problemática de la alfabetización en escritura académica nos permite observar y comprender lo que sucede en las distintas comunidades discursivas, pero no basta, es necesario volver la mirada hacia lo didáctico, para que así nuestros estudiantes puedan responder a los desafíos que les impone la formación en las distintas disciplinas a la hora de dar cuenta del conocimiento mediante los variados escritos propios de la comunidad a la que pertenecen. Si el conocimiento de las disciplinas debe aprenderse, estamos ciertos que lo mismo se aplica a la escritura en una suerte de simbiosis entre ambos. 


\section{REFERENCIAS}

Barton, D., Hamilton, M. e Ivanovic, R. (Eds.) (2000). Situated literacies. Reading and writing in context.Londres: Routledge.

Buendía, L., Colás, P. y Hernández, F. (1998). Métodos de Investigación en Psicopedagogía. Madrid: McGraw-Hill.

Camps, A. (1997). Escribir. La enseñanza y el aprendizaje de la composición escrita. Teoría y práctica de la Educación, 20, 24-33.

Carlino, P. (2013). Alfabetización académica diez años después. Revista Mexicana de Investigación Educativa, 18(57), 355-381.

Larrosa, J. (2001). Lenguaje y Educación. Revista Brasileira de Educação, 16, 6880.

Lea, M. y Street, B. (2006). The academic literacies model: Theory and applications. Theory into Practice, 45(4), 368-377.

Lemke, J. L (1990). Talking Science: Language, Learning, and Values. New Jersey: Ablex Publishing Corporation.

Li, J. y Klahr, D. (2006). The Psychology of Scientific Thinking: Implications for Science Teaching and Learning. En J. Rothony, P. Shane (Eds.), Teaching Science in the 21st Century (pp. 307-328). Arlington, VA: NSTA Press.

Marinkovich, J. y Córdova, A. (2014). La escritura en la Universidad: Objeto de estudio, método y discursos. Revista Signos, 47(84), 40-63.

Marinkovich, J. y Poblete, C. (2014). Alfabetización en escritura académica en Biología: propósitos comunicativos y niveles de alfabetización. ONOMÁZEIN, 30, 269-285.

Marinkovich, J., Velásquez, M. y Córdova, A. (Eds.). (2012). Comunidades académicas y culturas escritas: Construcciones discursivas desde las Ciencias y las Humanidades. Valparaíso: Ediciones Universitarias de Valparaíso.

Marinkovich, J., Velásquez, M., y Olivares, M. G. (2013). Niveles de alfabetización en escritura académica: de la reproducción a la transformación del conocimiento disciplinar. En N. Pardo; D. García; T. Oteíza y M. C. Asqueta (Comps.), Estudios del discurso en América Latina. Homenaje a Anamaría Harvey (pp. 129-149). Bogotá: ALED.

Monfort, N., Pagès, J. y Santisteban, A. (2011). ¿Cómo evaluar el pensamiento histórico del alumnado? En P. Miralles, S. Molina y A. Santisteban (Coords.), La evaluación en el proceso de enseñanza y aprendizaje de las ciencias sociales (Vol. 1) (pp. 221-232). Murcia: AUPDCS.

Paul, R. y Elder, L. (2006). The miniature guide to critical thinking: concepts and tools. Dillon Beach, C.A.: The Foundation for Critical Thinking.

Plá, S. (2005). Aprender a pensar históricamente. Madrid: Plaza y Valdés.

Porta, L. y Silva, M. (2003). La investigación cualitativa en el contexto educativo.

Tesis Doctoral. Argentina: Universidad Nacional de la Patagonia Austral. 
PUCV. (2015a). Licenciatura en Historia con mención en Ciencia Política. Recuperado de http://www.ucv.cl/pucv/pregrado/facultad-de-filosofia-y-educacion/licenciatura-en-historia-con-mencion-en-ciencia-politica/2015-06-11/143149. html.

PUCV. (2015b). Licenciatura en Biología. Recuperado de http://pucv.cl/pucv/ pregrado/facultad-de-ciencias/licenciatura-en-biologia/2015-06-08/173605. html.

Radloff, A. y De la Harpe, B. (2000). Helping students develop their writing skills - a resource for lecturers. En L. Richardson y J. Lidstone (Eds.), Flexible Learning for a Flexible Society (pp. 566-573). Toowoomba, Australia: ASET and HERDSA. Recuperado de http://www.ascilite.org/conferences/aset-archives/ confs/aset-herdsa2000/procs/radloff-a.html.

Romero, F. (2000). La escritura en los universitarios. Ciencias Humanas, 21. Recuperado de http://www.utp.edu.co/ - chumanas/revistas/revistas/rev21/romero.htm.

Ruiz, J. (2003). Metodología de la investigación cualitativa. Bilbao: Universidad de Deusto.

Santiuste, V. (Coord.), Ayala, C., Barrigüete, C., García, E., González, J., Rossignoli, J. y Toledo, E. (2001). El pensamiento crítico en la práctica educativa. Madrid: Fugaz Ediciones.

Sáiz, J. y López-Facal, R. (2015). Competencias y narrativas históricas: el pensamiento histórico de estudiantes y futuros profesores españoles de educación secundaria. Revista de Estudios Sociales, 52, 87-101.

Stake, R. (1995). The art of case study research. Thousand Oaks, C. A: Sage. 